\title{
Witt equivalence of function fields of conics
}

\author{
P. Gładki and M. Marshall \\ Communicated by A. P. Petravchuk
}

\begin{abstract}
A BSTRACT. Two fields are Witt equivalent if, roughly speaking, they have the same quadratic form theory. Formally, that is to say that their Witt rings of symmetric bilinear forms are isomorphic. This equivalence is well understood only in a few rather specific classes of fields. Two such classes, namely function fields over global fields and function fields of curves over local fields, were investigated by the authors in their earlier works [5] and [6]. In the present work, which can be viewed as a sequel to the earlier papers, we discuss the previously obtained results in the specific case of function fields of conic sections, and apply them to provide a few theorems of a somewhat quantitive flavour shedding some light on the question of numbers of Witt non-equivalent classes of such fields.
\end{abstract}

\section{Introduction}

One of the classical problems in bilinear algebra is to classify fields with respect to Witt equivalence, that is equivalence defined by isomorphism of their Witt rings of symmetric bilinear forms, which also includes fields of characteristic two. This problem is, in fact, manageable only when restricted to some specific classes of fields, which include trivial examples of quadratically closed fields, real closed fields, and finite fields, the case

Murray Marshall passed away in May 2015. Our community lost a brilliant mathematician and a wonderful man. We sorely miss him.

2000 MSC: Primary 11E81, 12J20; Secondary 11E04, 11E12.

Key words and phrases: symmetric bilinear forms, quadratic forms, Witt equivalence of fields, function fields, conic sections, valuations, Abhyankar valuations. 
of local fields ([13]), global fields ([16], [17], [18]), function fields in one variable over algebraically closed fields of characteristic $\neq 2$, and function fields in one variable over real closed fields ([7], [12]).

The authors of the present paper attempted to add two more classes of fields to this list, and investigated function fields over global fields [5] and function fields of curves over local fields [6], and managed to show that Witt equivalence of two function fields over global fields induces in a canonical way a bijection $v \leftrightarrow w$ between Abhyankar valuations $v$ of $K$ having residue field not finite of characteristic 2 and Abhyankar valuations $w$ of $L$ having residue field not finite of characteristic 2 ([5, Theorem 7.5]). Subsequently, a variant of this theorem has been also established in the local case ([6, Theorem 3.5]). Numerous corollaries providing some insight into the question of how Witt equivalence in these cases is behaved have been also drawn.

In the present paper we apply these results to take a closer look at the question of the number of Witt non-equivalent classes of function fields of conics, and provide some enumerative results in the case of conics defined over certain number fields. Some of them generalize in a certain way to the case of conics defined over arbitrary local fields. The main new results of the paper are found in Section 4. Throughout the entire exposition the authors use the language of hyperfields, which seem to provide a natural and convenient language to study Witt equivalence. We recall basic terminology and establish fundamental connections between hyperfields, valuations and quadratic forms in Section 2. All of this is a summary of Section 2 in [6], which, in turn, is a summary of Sections 2-6 in [5], and the reader more interested in all the technicalities is kindly referred to consult author's first paper [5] in the sequel. In Section 3 the authors prove a few additional facts on function fields of conics, and cite some old propositions that go back to Ernst Witt. The authors would like to believe that their results can be thought of as extensions of these beautiful, classical theorems by old masters.

\section{Hyperfields, valuations and Witt equivalence}

Hyperfields seem to provide a convenient and very natural way to describe Witt equivalence. In what follows we shall review the basic concepts and definitions used later in the paper. By a hyperfield we shall understand a system $(H,+, \cdot,-, 0,1)$, where $H$ is a set, + is a multivalued binary operation on $H$, i.e., a function from $H \times H$ to the set of all subsets 
of $H, \cdot$ is a binary operation on $H,-: H \rightarrow H$ is a function, and 0,1 are elements of $H$ such that

(I) $(H,+,-, 0)$ is a canonical hypergroup, i.e. for all $a, b, c \in H$,

(1) $c \in a+b \Rightarrow a \in c+(-b)$,

(2) $a \in b+0$ iff $a=b$,

(3) $(a+b)+c=a+(b+c)$,

(4) $a+b=b+a$;

(II) $(H, \cdot, 1)$ is a commutative monoid, i.e. for all $a, b, c \in H$,

(1) $(a b) c=a(b c)$,

(2) $a b=b a$,

(3) $a 1=a$;

(III) $a 0=0$ for all $a \in H$;

(IV) $a(b+c) \subseteq a b+a c$;

(V) $1 \neq 0$ and every non-zero element has a multiplicative inverse.

Hyperfields form a category with morphisms from $H_{1}$ to $H_{2}$, where $H_{1}, H_{2}$ are hyperfields, defined to be functions $\alpha: H_{1} \rightarrow H_{2}$ which satisfy $\alpha(a+b) \subseteq \alpha(a)+\alpha(b), \alpha(a b)=\alpha(a) \alpha(b), \alpha(-a)=-\alpha(a), \alpha(0)=0$, $\alpha(1)=1$. For a subgroup $T$ of $H^{*}$ (throughout the paper, for a set $A$, we shall always denote by $A^{*}$ the set $A \backslash\{0\}$ ), where $H$ is a hyperfield, denote by $H / m T$ the set of equivalence classes with respect to the equivalence relation $\sim$ on $H$ defined by

$$
a \sim b \text { if and only if } a s=b t \text { for some } s, t \in T .
$$

The operations on $H /{ }_{m} T$ are the obvious ones induced by the corresponding operations on $H$. Denote by $\bar{a}$ the equivalence class of $a$. Multiplication is defined in a natural way, and addition is set as follows:

$$
\bar{a} \in \bar{b}+\bar{c} \text { if and only if } a s \in b t+c u \text { for some } s, t, u \in T .
$$

$\left(H /{ }_{m} T,+, \cdot,-, 0,1\right)$ is then a hyperfield that we shall refer to as quotient hyperfield. For a hyperfield $H=(H,+, \cdot,-, 0,1)$ the prime addition on $H$ is defined by

$$
a+^{\prime} b= \begin{cases}a+b, & \text { if one of } a, b \text { is zero }, \\ a+b \cup\{a, b\}, & \text { if } a \neq 0, b \neq 0, b \neq-a, \\ H, & \text { if } a \neq 0, b \neq 0, b=-a .\end{cases}
$$

For any hyperfield $H:=(H,+, \cdot,-, 0,1), H^{\prime}:=\left(H,+^{\prime}, \cdot,-, 0,1\right)$ is also a hyperfield [5, Proposition 2.1]. We shall call $H^{\prime}$ the prime of the hyperfield 
$H$. The motivation for this definition comes from the following discussion: let $K$ be a field and define the quadratic hyperfield of $K$, denoted $Q(K)$, to be the prime of the hyperfield $K /{ }_{m} K^{* 2}$. Now let $W(K)$ be the Witt ring of non-degenerate symmetric bilinear forms over $K$; see [13], [14] or [20] for the definition in case $\operatorname{char}(K) \neq 2$ and [9], [11] or [15] for the definition in the general case. Recall that a (non-degenerate diagonal) binary form over $K$ is an ordered pair $\langle\bar{a}, \bar{b}\rangle, \bar{a}, \bar{b} \in K^{*} / K^{* 2}$, and its value set, denoted by $D_{K}\langle\bar{a}, \bar{b}\rangle$, is the set of non-zero elements of $\bar{a}+\bar{b}$. Now, a hyperfield isomorphism $\alpha$ between two quadratic hyperfields $Q(K)$ and $Q(L)$, where $K, L$ are fields, can be viewed as a group isomorphism $\alpha: K^{*} / K^{* 2} \rightarrow L^{*} / L^{* 2}$ such that $\alpha(-\overline{1})=-\overline{1}$ and

$$
\alpha\left(D_{K}\langle\bar{a}, \bar{b}\rangle\right)=D_{L}\langle\alpha(\bar{a}), \alpha(\bar{b})\rangle \text { for all } \bar{a}, \bar{b} \in K^{*} / K^{* 2} .
$$

Combining the results in [2], [8], and [14] one gets that two fields $K$ and $L$ are Witt equivalent, denoted $K \sim L$, iff $Q(K)$ and $Q(L)$ are isomorphic as hyperfields. Moreover, a morphism $\iota: H_{1} \rightarrow H_{2}$ between two hyperfields $H_{1}$ and $H_{2}$ induces a morphism $\bar{\iota}: H_{1} /{ }_{m} \Delta \rightarrow H_{2}$ where $\Delta:=\left\{x \in H_{1}^{*}: \iota(x)=1\right\}$. The morphism $\iota$ is said to be a quotient morphism if $\iota$ is an isomorphism, or, equivalently, if $\iota$ is surjective, and

$$
\iota(c) \in \iota(a)+\iota(b) \text { if and only if } c s \in a t+b u \text { for some } s, t, u \in \Delta .
$$

A morphism $\iota: H_{1} \rightarrow H_{2}$ is said to be a group extension if $\iota$ is injective, every $x \in H_{2}^{*} \backslash \iota\left(H_{1}^{*}\right)$ is rigid, meaning $1+x \subseteq\{1, x\}$, and

$$
\iota(1+y)=1+\iota(y), \text { for all } y \in H_{1}, y \neq-1 .
$$

For a field $K$ we adopt the standard notation from valuation theory: if $v$ is a valuation on $K, \Gamma_{v}$ denotes the value group, $A_{v}$ the valuation ring, $M_{v}$ the maximal ideal, $U_{v}$ the unit group, $K_{v}$ the residue field, and $\pi=\pi_{v}: A_{v} \rightarrow K_{v}$ the canonical homomorphism, i.e., $\pi(a)=a+M_{v}$. $v$ is discrete rank one if $\Gamma_{v}=\mathbb{Z}$. Denote $T=\left(1+M_{v}\right) K^{* 2}$. Consider the canonical group isomorphism $\alpha: U_{v} K^{* 2} /\left(1+M_{v}\right) K^{* 2} \rightarrow K_{v}^{*} / K_{v}^{* 2}$ induced by

$$
x \in U_{v} \mapsto \pi(x) \in K_{v}^{*} .
$$

and define $\iota: Q\left(K_{v}\right) \rightarrow K /{ }_{m} T$ by $\iota(0)=0$ and $\iota(a)=\alpha^{-1}(a)$ for $a \in K_{v}^{*} / K_{v}^{* 2}$. If $v$ is non-trivial, then the map $Q(K) \rightarrow K / m T$ defined by $\bar{x} \mapsto x T$ is a quotient morphism and $\iota$ is a group extension. The cokernel of the group embedding $\alpha^{-1}: K_{v}^{*} / K_{v}^{* 2} \rightarrow K^{*} / T$ is equal to $K^{*} / U_{v} K^{* 2} \cong \Gamma_{v} / 2 \Gamma_{v}$. We reflect this by calling $K / m T$ a group extension 
of $Q\left(K_{v}\right)$ by the group $\Gamma_{v} / 2 \Gamma_{v}$. If $v$ is non-trivial and $\operatorname{char}\left(K_{v}\right) \neq 2$, then $K / m T$ can be naturally identified with $Q\left(\tilde{K}_{v}\right)$, where $\tilde{K}_{v}$ denotes the henselization of $(K, v)$. If $v, v^{\prime}$ are valuations on $K$ with $v \preceq v^{\prime}$, i.e. such that $v^{\prime}$ is a coarsening of $v$, meaning $A_{v} \subseteq A_{v^{\prime}}$, then $M_{v^{\prime}} \subseteq M_{v}$, and, consequently, $\left(1+M_{v^{\prime}}\right) K^{* 2} \subseteq\left(1+M_{v}\right) K^{* 2}$. If we denote by $\bar{v}$ the valuation on $K_{v^{\prime}}$ induced by $v$, that is

$$
\bar{v}\left(\pi_{v^{\prime}}(a)\right)=v(a), \text { for } a \in U_{v^{\prime}},
$$

then the valuations $\bar{v}$ and $v$ have the same residue field. If $v$ and $v^{\prime}$ are non-trivial and $v^{\prime}$ is a proper coarsening of $v$, meaning $A_{v} \subsetneq A_{v^{\prime}}$, then $K / m\left(1+M_{v}\right) K^{* 2}$ is a group extension of the hyperfield $K_{v^{\prime}} / m\left(1+M_{\bar{v}}\right) K_{v^{\prime}}^{* 2}$ and the following diagram is commutative:

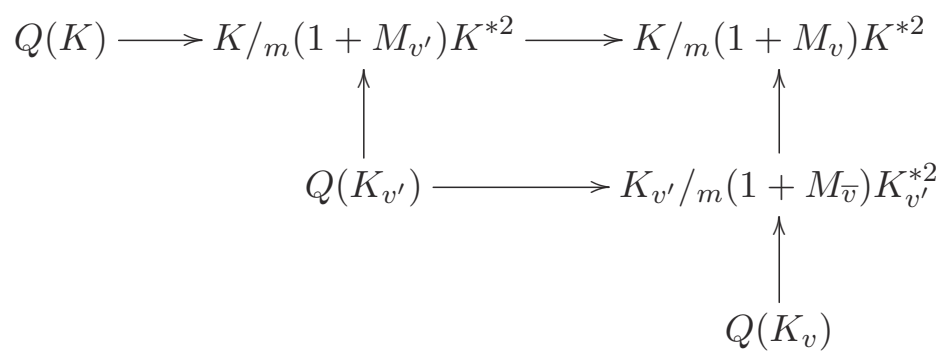

For a subgroup $T$ of $K^{*}$ we say that $x \in K^{*}$ is $T$-rigid if $T+T x \subseteq T \cup T x$, and denoting by

$$
B(T):=\left\{x \in K^{*}: \text { either } x \text { or }-x \text { is not } T \text {-rigid }\right\}
$$

we will refer to the elements of $B(T)$ as to the $T$-basic elements. If $x \in K^{*}$ is $T$-rigid and $y=t x$, for some $t \in T$, then $y$ is $T$-rigid, so that $B(T)$ is a union of cosets of $T$. If $\pm T=B(T)$, and either $-1 \in T$ or $T$ is additively closed, we shall say that the subgroup $T$ is exceptional. If $H \subseteq K^{*}$ is a subgroup containing $B(T)$, then there exists a subgroup $\hat{H}$ of $K^{*}$ such that $H \subseteq \hat{H}$ and $(\hat{H}: H) \leqslant 2$, and a valuation $v$ of $K$ such that $1+M_{v} \subseteq T$ and $U_{v} \subseteq \hat{H}$. Moreover, $\hat{H}$ can be taken to be simply $H$, unless $T$ is exceptional [1, Theorem 2.16]. $B\left(K^{* 2}\right)$ is a subgroup of $K^{*}$, and in the case when $T=\left(1+M_{v}\right) K^{* 2}$, for some non-trivial valuation $v$ of $K, B(T) \subseteq U_{v} K^{* 2}$ and

$$
B(T)=\left\{x \in K^{*}: \bar{x}=\iota(\bar{y}) \text { for some } y \in B\left(K_{v}^{* 2}\right)\right\},
$$

where $\iota: Q\left(K_{v}\right) \hookrightarrow K / m T$ is the morphism described above. $B(T)$ is a group and the group isomorphism $\iota: K_{v}^{*} / K_{v}^{* 2} \rightarrow U_{v} K^{* 2} / T$ induces a 
group isomorphism $B\left(K_{v}^{* 2}\right) / K_{v}^{* 2} \rightarrow B(T) / T . T$ is exceptional if and only if $K_{v}^{* 2}$ is exceptional. The main result that explains how valuations in Witt equivalent fields are matched is the following one:

Theorem 2.1 ([5, Theorem 5.3]). Suppose $K, L$ are fields, $\alpha: Q(K) \rightarrow$ $Q(L)$ is a hyperfield isomorphism and $v$ is a valuation on $K$ such that $\Gamma_{v}$ is finitely generated as an abelian group. Suppose either (i) the basic part of $\left(1+M_{v}\right) K^{* 2}$ is $U_{v} K^{* 2}$ and $\left(1+M_{v}\right) K^{* 2}$ is unexceptional, or (ii) the basic part of $\left(1+M_{v}\right) K^{* 2}$ is $\left(1+M_{v}\right) K^{* 2}$ and $\left(1+M_{v}\right) K^{* 2}$ has index 2 in $U_{v} K^{* 2}$. Then there exists a valuation $w$ on $L$ such that the image of $\left(1+M_{v}\right) K^{* 2} / K^{* 2}$ under $\alpha$ is $\left(1+M_{w}\right) L^{* 2} / L^{* 2}$ and $\left(L^{*}: U_{w} L^{* 2}\right) \geqslant\left(K^{*}\right.$ : $U_{v} K^{* 2}$ ). If (i) holds, then the image of $U_{v} K^{* 2} / K^{* 2}$ under $\alpha$ is $U_{w} L^{* 2} / L^{* 2}$.

If $K, L$ are fields, $v$ and $w$ are non-trivial, and $\alpha: Q(K) \rightarrow Q(L)$ is a hyperfield isomorphism such that the image of $\left(1+M_{v}\right) K^{* 2} / K^{* 2}$ under $\alpha$ is $\left(1+M_{w}\right) L^{* 2} / L^{* 2}$, then $\alpha$ induces a hyperfield isomorphism $K / m\left(1+M_{v}\right) K^{* 2} \rightarrow L / m\left(1+M_{w}\right) L^{* 2}$ such that the diagram

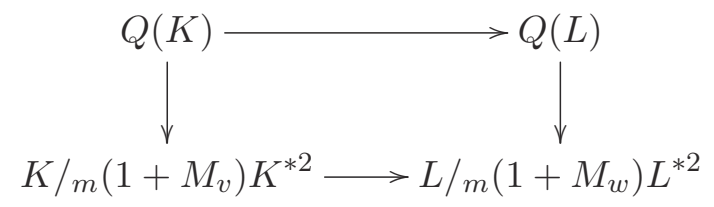

commutes. If, in addition, the image of $U_{v} K^{* 2} / K^{* 2}$ under $\alpha$ is $U_{w} L^{* 2} / L^{* 2}$, then $\alpha$ induces a hyperfield isomorphism $Q\left(K_{v}\right) \rightarrow Q\left(L_{w}\right)$ and a group isomorphism $\Gamma_{v} / 2 \Gamma_{v} \rightarrow \Gamma_{w} / 2 \Gamma_{w}$ such that the diagrams

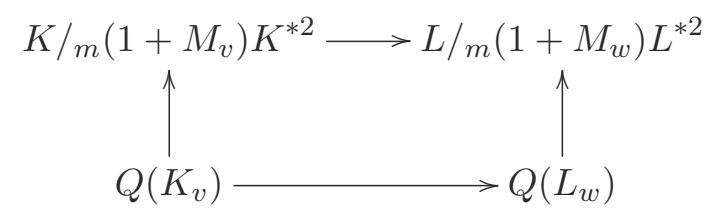

and

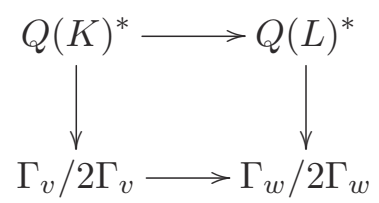

both commute.

Recall that the nominal transcendence degree of $K$ is defined to be

$$
\operatorname{ntd}(K):=\left\{\begin{array}{ll}
\operatorname{trdeg}(K: \mathbb{Q}) & \text { if } \operatorname{char}(K)=0 \\
\operatorname{trdeg}\left(K: \mathbb{F}_{p}\right)-1 & \text { if } \operatorname{char}(K)=p \neq 0
\end{array} .\right.
$$


For an abelian group $\Gamma$, its rational rank of $\Gamma$, denoted $\operatorname{rk}_{\mathbb{Q}}(\Gamma)$, is defined to be the dimension of the $\mathbb{Q}$-vector space $\Gamma \otimes_{\mathbb{Z}} \mathbb{Q}$. If $K$ is a function field over $k$ and $v$ is a valuation on $K$, the Abhyankar inequality asserts that

$$
\operatorname{trdeg}(K: k) \geqslant \operatorname{rk}_{\mathbb{Q}}\left(\Gamma_{v} / \Gamma_{v \mid k}\right)+\operatorname{trdeg}\left(K_{v}: k_{v \mid k}\right),
$$

where $v \mid k$ denotes the restriction of $v$ to $k$. The valuation $v$ is Abhyankar (relative to $k$ ) if the actual equality holds in the Abhyankar inequality, that is

$$
\operatorname{trdeg}(K: k)=\operatorname{rk}_{\mathbb{Q}}\left(\Gamma_{v} / \Gamma_{v \mid k}\right)+\operatorname{trdeg}\left(K_{v}: k_{v \mid k}\right) .
$$

In this case it is known that $\Gamma_{v} / \Gamma_{v \mid k}$ is finitely generated and $K_{v}$ is a function field over $k_{v \mid k}$.

\section{Function fields of conics}

Let $k$ be a field of characteristic $\neq 2$.

Proposition 3.1. Let $a, b \in k^{*}$ and assume that $a x^{2}+b y^{2}=1$ has no $k$-rational points. Then $\frac{k[x, y]}{\left(a x^{2}+b y^{2}-1\right)}$ is a principal ideal domain.

Proof. This is well known, see [4].

Set $k_{a, b}:=\mathrm{qf} \frac{k[x, y]}{\left(a x^{2}+b y^{2}-1\right)}$, the quotient field of $\frac{k[x, y]}{\left(a x^{2}+b y^{2}-1\right)}$. We assume always that $a, b \in k^{*}$.

Proposition 3.2. The field of constants of $k_{a, b}$ over $k$ is equal to $k$.

Proof. Clearly $k_{a, b}=k(x)\left(\sqrt{\frac{1-a x^{2}}{b}}\right)$. Suppose $f=f_{0}+f_{1} \sqrt{\frac{1-a x^{2}}{b}}, f_{0}, f_{1} \in$ $k(x)$, is algebraic over $k$. Then $\bar{f}=f_{0}-f_{1} \sqrt{\frac{1-a x^{2}}{b}}$ is also algebraic over $k$. Consequently, $f_{0}=(f+\bar{f}) / 2$ and $f_{0}^{2}-f_{1}^{2}\left(\frac{1-a x^{2}}{b}\right)=f \bar{f}$ are algebraic over $k$. It follows that $f_{1}^{2}\left(\frac{1-a x^{2}}{b}\right)$ is algebraic over $k$, i.e., $f_{1}=0$, and $f_{0} \in k$.

For $a, b \in k^{*},\left(\frac{a, b}{k}\right)$ denotes the quaternion algebra over $k$, i.e., the 4-dimension central simple algebra over $k$ generated by $i, j$ subject to $i^{2}=a, j^{2}=b, j i=-i j$. We identify quaternion algebras over $k$ which are isomorphic as $k$-algebras, equivalently, are equal as elements of the Brauer group of $k$.

Proposition 3.3. The following are equivalent:

(1) $\left(\frac{a, b}{k}\right)=1$ (i.e., $\left(\frac{a, b}{k}\right)$ splits over $k$ ).

(2) $\langle 1,-a\rangle \otimes\langle 1,-b\rangle \sim 0$ over $k$. 
(3) $1 \in D_{k}\langle a, b\rangle$.

(4) The conic $a x^{2}+b y^{2}=1$ has a rational point.

(5) $k_{a, b}$ is purely transcendental over $k$.

Proof. The equivalence of (1), (2), (3) and (4) is well-known from quadratic form theory. If $(p, q)$ is a rational point of $a x^{2}+b y^{2}=1$ then $k_{a, b}=k(z)$ where $z:=\frac{y-q}{x-p}$. Conversely, if $k_{a, b}=k(z)$ then, choosing $f(z), g(z), h(z) \in$ $k[z]$ so that $x=\frac{f(z)}{h(z)}, y=\frac{g(z)}{h(z)}$, and choosing $r \in k$ so that $h(r) \neq 0$, one sees that $\left(\frac{f(r)}{h(r)}, \frac{g(r)}{h(r)}\right)$ is a rational point of $a x^{2}+b y^{2}=1$. Note: This argument fails if $|k|<\infty$, but the conclusion continues to hold even in this case, since $|k|<\infty \Rightarrow$ the quadratic form $\langle a, b\rangle$ is $k$-universal.

From the definition of $k_{a, b}$ it is clear that $1 \in D_{k_{a, b}}\langle a, b\rangle$, so $\left(\frac{a, b}{k}\right)$ splits over $k_{a, b}$. Of course, 1 also splits over $k_{a, b}$ (since it splits over $k$ ). Conversely one has the following:

Proposition 3.4 (E. Witt). The only quaternion algebras defined over $k$ which split over $k_{a, b}$ are $\left(\frac{a, b}{k}\right)$ and 1 .

Proof. See [19, Satz, Page 465] or [10, Lemma 4.4].

We write $K \cong_{k} L$ to indicate that the field extensions $K, L$ of $k$ are $k$-isomorphic.

Proposition 3.5 (E. Witt). The following are equivalent:

(1) $\left(\frac{a, b}{k}\right)=\left(\frac{c, d}{k}\right)$.

(2) $k_{a, b} \cong_{k} k_{c, d}$.

Proof. Then implication (1) $\Rightarrow(2)$ is [19, Satz, page 464]. The implication $(2) \Rightarrow(1)$ is immediate from the Proposition 3.4.

We will need to know which orderings of $k$ extend to $k_{a, b}$.

Lemma 3.6. An ordering $<$ on $k$ extends to an ordering on $k_{a, b}$ iff at least one of $a, b$ is positive at $<$.

Proof. One way is clear. If $<$ extends to $k_{a, b}$ then, in $k_{a, b}, a x^{2}+b y^{2}=1>0$ so at least one of $a, b$ must be positive. Conversely, suppose at least one if $a, b$ is positive. Fix a real closure $R$ of $(k,<)$. Clearly $\exists \bar{x}, \bar{y} \in R$ satisfying $a \bar{x}^{2}+b \bar{y}^{2}=1$. Then $\left(\frac{a, b}{k}\right)$ splits over $k(\bar{x}, \bar{y})$ and hence also over $R$, so $k_{a, b} \hookrightarrow R_{a, b} \equiv R(t)$. Any one of the infinitely many orderings of $R(t)$ extends the ordering $<$. 


\section{Application to function fields of conics}

Let $K$ be a function field in one variable (i.e. a function field $K$ over $k$ satisfying $\operatorname{trdeg}(K: k)=1$ ) over a global field $k$ (we do not assume that $k$ is the field of constants of $K$ over $k$, i.e. the algebraic closure of $k$ in $K$ ). We consider the set $\nu_{K}$ consisting of all non-trivial Abhyankar valuations of $K$ over $k$ such that $K_{v}$ is not finite of characteristic 2 . Thus

$$
\nu_{K}=\nu_{K, 0} \cup \nu_{K, 1} \cup \nu_{K, 2} \cup \nu_{K, 3} \cup \nu_{K, 4} \text { (disjoint union) }
$$

where $\nu_{K, 0}$ is the set of valuations $v$ of $K$ such that $\Gamma_{v}=\mathbb{Z}$ and $K_{v}$ is a number field, $\nu_{K, 1}$ is the set of valuations $v$ on $K$ such that $\Gamma_{v}=\mathbb{Z}$ and $K_{v}$ is a global field of characteristic $p \neq 0,2, \nu_{K, 2}$ is the set of valuations $v$ on $K$ such that $\Gamma_{v}=\mathbb{Z}$ and $K_{v}$ is a global field of characteristic $2, \nu_{K, 3}$ is the set of valuations $v$ on $K$ such that $\Gamma_{v}=\mathbb{Z} \times \mathbb{Z}, K_{v}$ is a finite field of characteristic $\neq 2$ and $-1 \notin K_{v}^{* 2}$ and $\nu_{K, 4}$ is the set of valuations $v$ on $K$ such that $\Gamma_{v}=\mathbb{Z} \times \mathbb{Z}, K_{v}$ is a finite field of characteristic $\neq 2$ and $-1 \in K_{v}^{* 2}$.

Of course, some of the sets $\nu_{K, i}$ may be empty. Specifically, if $\operatorname{char}(K) \notin$ $\{0,2\}$ then $\nu_{K, i}=\varnothing$ for $i \in\{0,2\}$ and if $\operatorname{char}(K)=2$ then $\nu_{K, i}=\varnothing$ for $i \in\{0,1,3,4\}$.

Corollary 4.1 ([5, Corollary 8.1]). Suppose $K, L$ are function fields in one variable over global fields which are Witt equivalent via a hyperfield isomorphism $\alpha: Q(K) \rightarrow Q(L)$. Then for each $i \in\{0,1,2,3,4\}$ there is a uniquely defined bijection between $\nu_{K, i}$ and $\nu_{L, i}$ such that, if $v \leftrightarrow w$ under this bijection, then $\alpha$ maps $\left(1+M_{v}\right) K^{* 2} / K^{* 2}$ onto $\left(1+M_{w}\right) L^{* 2} / L^{* 2}$ and $U_{v} K^{* 2} / K^{* 2}$ onto $U_{w} L^{* 2} / L^{* 2}$ for $i \in\{0,1,2,3\}$ and such that $\alpha$ maps $\left(1+M_{v}\right) K^{* 2} / K^{* 2}$ onto $\left(1+M_{w}\right) L^{* 2} / L^{* 2}$ for $i=4$.

Corollary 4.2 ([5, Corollary 8.2]). Let $K \sim L$ be function fields in one variable over global fields $k, \ell$ respectively, with fields of constants $k$ and $\ell$ respectively. Then $k \sim \ell$ except possibly in the case where $k, \ell$ are both number fields. In the latter case assume there exists $v \in \nu_{K, 0}$ with $K_{v}=k$ and $w \in \nu_{L, 0}$ with $L_{w}=\ell$. Then $k \sim \ell$.

Remark 4.3. The last assertion of Corollary 4.2 applies, in particular, in the case where $K=k(x), L=\ell(x)$, where $k, \ell$ are number fields.

Let $k$ be a number field. Every ordering of $k$ is archimedean, so that it corresponds to a real embedding $k \hookrightarrow \mathbb{R}$. Denote by $r_{1}$ the number of 
real embeddings of $k$ and by $r_{2}$ the number of conjugate pairs of complex embeddings of $k$. Then $[k: \mathbb{Q}]=r_{1}+2 r_{2}$. Furthermore, let

$$
V_{k}:=\left\{r \in k^{*}:(r)=\mathfrak{a}^{2} \text { for some fractional ideal } \mathfrak{a} \text { of } k\right\} .
$$

$V_{k}$ is a subgroup of $k^{*}$ and $k^{* 2} \subseteq V_{k}$. We will need the following result, which is a version of [5, Theorem 8.6]:

Theorem 4.4. Suppose $K$ and $L$ are function fields of genus zero curves over number fields with fields of constants $k$ and $\ell$ respectively, and $\alpha: Q(K) \rightarrow Q(L)$ is a hyperfield isomorphism. Then

(1) $r \in k^{*} / k^{* 2}$ iff $\alpha(r) \in \ell^{*} / \ell^{* 2}$.

(2) $\alpha$ induces a bijection between orderings $P$ of $k$ which extend to $K$ and orderings $Q$ of $\ell$ which extend to $L$ via $P \leftrightarrow Q$ iff $\alpha$ maps $P^{*} / k^{* 2}$ to $Q^{*} / \ell^{* 2}$.

(3) $\alpha$ maps $V_{k} / k^{* 2}$ to $V_{\ell} / \ell^{* 2}$.

$(4)[k: \mathbb{Q}]=[\ell: \mathbb{Q}]$.

(5) $K$ is purely transcendental over $k$ iff $L$ is purely transcendental over $\ell$. In this case, the map $r \mapsto \alpha(r)$ defines a hyperfield isomorphism between $Q(k)$ and $Q(\ell)$, and the 2-ranks of the ideal class groups of $k$ and $\ell$ are equal.

First part the proof follows the same line of reasoning as the proof of [5, Theorem 8.6], but we shall provide it here for the sake of the completeness of the exposition. We will need two lemmas:

Lemma 4.5 ([5, Lemma 8.4]). The 2-rank of the group $V_{k} / k^{* 2}$ is $r_{1}+r_{2}+t$ where $t$ is the 2-rank of the ideal class group of $k$.

Lemma 4.6 (see also [5, Lemma 8.5]). Suppose $v$ is a discrete rank 1 valuation on $k$, and $a, b \in k^{*}$. There exists an Abhyankar extension of $v$ to $k_{a, b}$ such that $v\left(k_{a, b}{ }^{*}\right)=v\left(k^{*}\right)$.

Proof. Denote by $\hat{k}=\hat{k}_{v}$ the completion of $k$ at $v$. Suppose first that $\left(\frac{a, b}{k}\right)$ splits over $\hat{k}$. Then $k_{a, b} \hookrightarrow \hat{k}_{a, b}=\hat{k}(x)$. Extend the valuation $v$ to $\hat{k}(x)$ by defining $v\left(\sum_{i=0}^{n} a_{i} x^{i}\right)=\min \left\{v\left(a_{i}\right): 0 \leqslant i \leqslant n\right\}$ and $v\left(\frac{f(x)}{g(x)}\right)=$ $v(f(x))-v(g(x))$.

Suppose now that $\left(\frac{a, b}{k}\right)$ does not split over $\hat{k}$. Choose $c \in \hat{k}^{*} \backslash \hat{k}^{* 2}$ so that $\hat{k}(\sqrt{c})$ is the unique unramified quadratic extension of $\hat{k}$. The group $D_{\hat{k}}\langle 1,-c\rangle$ has index 2 in $\hat{k}^{*}$, so $\exists d \in \hat{k}^{*}$ such that $\left(\frac{c, d}{\hat{k}}\right) \neq 1$. Since there is exactly one non-split quaternion algebra over $\hat{k}$, we see that $\left(\frac{a, b}{\hat{k}}\right)=\left(\frac{c, d}{\hat{k}}\right)$. Thus $\left(\frac{a, b}{k}\right)$ splits over $\hat{k}(\sqrt{c})$ and we can proceed as before, but with $\hat{k}$ replaced by $\hat{k}(\sqrt{c})$. 
We now proceed to the proof of Theorem 4.4.

Proof. By our hypothesis, $K=k_{a, b}$ for some $a, b \in k^{*}$ and $L=\ell_{c, d}$ for some $c, d \in \ell^{*}$. If $r \in k^{*} / k^{* 2}$, then $r \in U_{v} K^{* 2} / K^{* 2}$ for all $v \in \nu_{K, 0}$. This implies $\alpha(r) \in U_{w} L^{* 2} / L^{* 2}$ for all $w \in \nu_{L, 0}$. Since $\frac{\ell[x, y]}{\left(c x^{2}+d y^{2}-1\right)}$ is a principal ideal domain with unit group $\ell^{*}$ this implies, in turn, that $\alpha(r) \in \ell^{*} / \ell^{* 2}$. Thus $\alpha$ induces a group isomorphism between $k^{*} / k^{* 2}$ and $\ell^{*} / \ell^{* 2}$. This proves (1). Suppose $P$ is an ordering of $k$ which extends to an ordering $P_{1}$ of $K$. Since $\alpha$ is a hyperfield isomorphism, there exists a unique ordering $Q_{1}$ of $L$ such that $\alpha\left(P_{1}^{*} / K^{* 2}\right)=Q_{1}^{*} / L^{* 2}$. Denote by $Q$ the restriction of $Q_{1}$ to $\ell$. Clearly $\alpha\left(P^{*} / k^{* 2}\right)=Q^{*} / \ell^{* 2}$. This proves (2). Lemma 4.6 implies that

$$
V_{k} / k^{* 2}=\left\{r \in k^{*} / k^{* 2}: r \in U_{v} K^{* 2} / K^{* 2} \forall v \in \nu_{K, 1} \cup \nu_{K, 2}\right\}
$$

so (3) is clear. Observe that if $v \leftrightarrow w, v \in \nu_{K, 0}, w \in \nu_{L, 0}$, the diagram

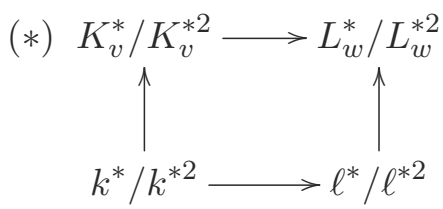

is commutative. The vertical arrows are the maps induced by the field embeddings $k \hookrightarrow K_{v}, \ell \hookrightarrow L_{w}$. Since the top arrow in $(*)$ defines a hyperfield isomorphism between $Q\left(K_{v}\right)$ and $Q\left(L_{w}\right)$ we know that $\left[K_{v}\right.$ : $\mathbb{Q}]=\left[L_{w}: \mathbb{Q}\right]$. Choose $v, w$ with $\left[K_{v}: \mathbb{Q}\right]=\left[L_{w}: \mathbb{Q}\right]$ minimal. If $\left(\frac{a, b}{k}\right)$ splits over $k$ then $K_{v}=k$ and $\left[K_{v}: \mathbb{Q}\right]=[k: \mathbb{Q}]$. If $\left(\frac{a, b}{k}\right)$ is non-split over $k$ then $\left(\frac{a, b}{k}\right)$ splits over a quadratic extension, so $\left[K_{v}: k\right]=2$, i.e., $\left[K_{v}: \mathbb{Q}\right]=2[k: \mathbb{Q}]$. Thus we see that either $[k: \mathbb{Q}]=[\ell: \mathbb{Q}]$, $2[k: \mathbb{Q}]=[\ell: \mathbb{Q}]$, or $[k: \mathbb{Q}]=2[\ell: \mathbb{Q}]$. Suppose $2[k: \mathbb{Q}]=[\ell: \mathbb{Q}]$, i.e., $\left[K_{v}: \mathbb{Q}\right]=2$, and $L_{w}=\ell$. Then the left vertical arrow in $(*)$ has a non-trivial kernel, but the right vertical arrow in $(*)$ is an isomorphism, a contradiction. Thus $2[k: \mathbb{Q}]=[\ell: \mathbb{Q}]$ is impossible. A similar argument shows that $[k: \mathbb{Q}]=2[\ell: \mathbb{Q}]$ is impossible. This proves $(4)$. If $\left(\frac{a, b}{k}\right)$ is nonsplit and $\left(\frac{c, d}{\ell}\right)$ is split, then $\left[K_{v}: k\right]=2$ and $L_{w}=\ell$ so $2[k: \mathbb{Q}]=[\ell: \mathbb{Q}]$, contradicting what was proved in (4). This proves the first statement of (5). If $\left(\frac{a, b}{k}\right)$ and $\left(\frac{c, d}{\ell}\right)$ are both split then $k=K_{v}, \ell=L_{w}$. In this case it follows from the commutativity of $(*)$ and what was already proved that the map $r \mapsto \alpha(r)$ defines a hyperfield isomorphism between $Q(k)$ and $Q(\ell)$. Since it is well-known that $r_{1}$ and $r_{2}$ are invariant under Witt equivalence, the 
last assertion of (5) is immediate now, from (3) and Lemma 4.5. This completes the proof.

Question 1: Is it true that every hyperfield isomorphism $\alpha: Q\left(k_{a, b}\right) \rightarrow$ $Q\left(\ell_{c, d}\right)$ induces a hyperfield isomorphism between $Q(k)$ and $Q(\ell)$ ? I.e., is the hypothesis that $\left(\frac{a, b}{k}\right)$ and $\left(\frac{c, d}{\ell}\right)$ are split really necessary?

Question 2: For a given number field $k$, are there infinitely many Witt inequivalent fields of the form $k_{a, b}, a, b \in k^{*}$ ? All we are able to prove in this regard is the following:

Theorem 4.7. Let $k$ be a number field, $r=$ the number of orderings of $k, w=$ the number of Witt inequivalent fields of the form $k_{a, b}, a, b \in k^{*}$. Then

$$
w \geqslant\left\{\begin{array}{l}
2 \text { if }-1 \in D_{k}\langle 1,1\rangle, \\
3 \text { if }-1 \notin D_{k}\langle 1,1\rangle, k \text { is not formally real, } \\
r+3 \text { if } k \text { is formally real. }
\end{array}\right.
$$

Proof. For a prime $\mathfrak{p}$ of $k$ (finite or infinite), denote by $\hat{k}_{\mathfrak{p}}$ the completion of $k$ at $\mathfrak{p}$.

Case $1:-1 \in D_{k}\langle 1,1\rangle$. Fix $a, b \in k^{*}$ so that $\left(\frac{a, b}{k}\right)$ does not split over $k$. E.g., fix some finite prime $\mathfrak{p}$ of $k$ and choose $a, b \in k^{*}$ so that $a \notin \hat{k}_{\mathfrak{p}}^{* 2}$ and $b \notin D_{\hat{k}_{\mathrm{p}}}\langle 1,-a\rangle$. Theorem $4.4(5)$ implies that $k_{a, b} \nsim k_{1,1}$.

Case 2 : $-1 \notin D_{k}\langle 1,1\rangle, k$ not formally real. By hypothesis, $\left(\frac{-1,-1}{k}\right)$ is not split over $k$. By the Hasse norm theorem there exists a finite prime $\mathfrak{p}$ such that $\left(\frac{-1,-1}{k}\right)$ is not split over $\hat{k}_{\mathfrak{p}}$. By Hilbert reciprocity there exists a finite prime $\mathfrak{q} \neq \mathfrak{p}$ such that $\left(\frac{-1,-1}{k}\right)$ is not split over $\hat{k}_{\mathfrak{q}}$. Fix $b \in k^{*}$ so that $b$ is sufficiently close to -1 at $\mathfrak{p}$ and $b$ is sufficiently close to 1 at $\mathfrak{q}$. Then $b$ is minus a square in $\hat{k}_{\mathfrak{p}}$ and $b$ is a square in $\hat{k}_{\mathfrak{q}}$. If there exists a hyperfield isomorphism $\alpha: Q\left(k_{-1,-1}\right) \rightarrow Q\left(k_{b,-1}\right)$ then, since $\left(\frac{-1,-1}{k}\right)$ splits over $k_{-1,-1}$ and $\alpha(-1)=-1$, it follows that $\left(\frac{-1,-1}{k}\right)$ splits over $k_{b,-1}$. According to Proposition 3.4, this implies $\left(\frac{-1,-1}{k}\right)=1$ or $\left(\frac{-1,-1}{k}\right)=\left(\frac{b,-1}{k}\right)$, i.e., $\left(\frac{-1,-1}{k}\right)=1$ or $\left(\frac{-b,-1}{k}\right)=1$. Since $\left(\frac{-1,-1}{k}\right)$ does not split over $\hat{k}_{\mathfrak{p}}$ and $\left(\frac{-b,-1}{k}\right)$ does not split over $\hat{k}_{\mathfrak{q}}$ this is a contradiction. Thus $k_{-1,-1} \not k_{b,-1}$. Since $\left(\frac{-1,-1}{k}\right) \neq 1$ and $\left(\frac{b,-1}{k}\right) \neq 1$ one also has that $k_{-1,-1} \not k_{1,1}$ and $k_{b,-1} \not k_{1,1}$.

Case 3: Suppose $k$ is formally real. For each integer $0 \leqslant i \leqslant r$ choose $a_{i} \in k^{*}$ so that $a_{i}>0$ for exactly $i$ of the orderings of $k$. Without loss of generality, $a_{0}=-1, a_{r}=1$. By Lemma 3.6 exactly $i$ of the orderings of 
$k$ extend to $k_{a_{i},-1}$, so $k_{a_{i},-1} \not k_{a_{j},-1}$ if $i \neq j$ by Theorem 4.4 (2). This proves $w \geqslant r+1$. Fix $0 \leqslant i \leqslant r$ so that $r-i$ is odd (e.g., take $i=r-1$ ). By Hilbert reciprocity there exists a finite prime $\mathfrak{p}$ of $k$ such that $\left(\frac{a_{i},-1}{k}\right)$ does not split over $\hat{k}_{\mathfrak{p}}$. Pick $\bar{a}_{i} \in k^{*}$ sufficiently close to 1 at $\mathfrak{p}$ and sufficiently close to $a_{i}$ at each ordering of $k$. Then $\bar{a}_{i}$ is a square in $\hat{k}_{\mathfrak{p}}$ and has the same sign as $a_{i}$ at each ordering. By Hilbert reciprocity there exists a finite prime $\mathfrak{q} \neq \mathfrak{p}$ so that $\left(\frac{\bar{a}_{i},-1}{k}\right)$ does not split over $\hat{k}_{\mathfrak{q}}$. Pick $b_{r} \in k^{*}$ so that $b_{r}$ is close to $a_{r}(=1)$ at each ordering of $k$ and such that $b_{r}$ is close to $a_{i}$ at $\mathfrak{p}$. Then $k_{b_{r},-1} \not k_{a_{r},-1}$, by Theorem 4.4 (5) (because $\left(\frac{a_{r},-1}{k}\right)$ splits over $k$ but $\left(\frac{b_{r},-1}{k}\right)$ does not split over $\left.\hat{k}_{\mathfrak{p}}\right)$. Also, all orderings of $k$ extend to $k_{b_{r},-1}$, so $k_{b_{r},-1} \not k_{a_{j},-1}$ for $0 \leqslant j<r$, by Theorem 4.4 (2). Finally, pick $b_{0} \in k^{*}$ so that $b_{0}$ is close to $a_{0}(=-1)$ at each ordering of $k, b_{0}$ is close to $a_{i}$ at $\mathfrak{p}$ and $b_{0}$ is close to $-\bar{a}_{i}$ at $\mathfrak{q}$. A similar argument to that used in Case 2 shows that $k_{b_{0},-1} \not k_{-1,-1}$. Theorem $4.4(2)$ shows that $k_{b_{0},-1} \nsim k_{a_{j},-1}$ for $0<j \leqslant r$ and $k_{b_{0},-1} \nsim k_{b_{r},-1}$.

Question 3: For a fixed integer $n \geqslant 1$, are there infinitely many Witt inequivalent fields $k_{a, b}, k$ a number field, $[k: \mathbb{Q}]=n, a, b \in k^{*}$ ?

Following the argument of [5, Corollary 8.8] we are able to partially answer this question in case $n=2$ : let $d$ be a square free integer and denote by $N$ the number of prime integers that ramify in $\mathbb{Q}(\sqrt{d})$. This is equal to the number of prime divisors of the discriminant of $\mathbb{Q}(\sqrt{d})$. Recall that the discriminant of $\mathbb{Q}(\sqrt{d})$ is $d$ if $d \equiv 1 \bmod 4$ and $4 d$ otherwise. Then the 2-rank of the class group of $\mathbb{Q}(\sqrt{d})$ is

$$
\left\{\begin{array}{l}
N-2 \text { if } d>0 \text { and } d \notin D_{\mathbb{Q}}\langle 1,1\rangle, \\
N-1 \text { otherwise. }
\end{array}\right.
$$

See [3, Corollary 18.3] for the proof. In particular, there are infinitely many possible values for the 2-rank of the class number for fields of the sort $\mathbb{Q}(\sqrt{d}), d \in \mathbb{Q}^{*} \backslash \mathbb{Q}^{* 2}$. Combining this with Theorem 4.4 yields:

Corollary 4.8. There are infinitely many Witt inequivalent fields of the form $k(x), k$ a quadratic extension of $\mathbb{Q}$.

Finally, we consider an application of Corollary 4.1 and Theorem 4.4 to fields of the form $\mathbb{Q}_{a, b}$.

Proposition 4.9. Suppose $\alpha: Q\left(\mathbb{Q}_{a, b}\right) \rightarrow Q\left(\mathbb{Q}_{c, d}\right)$ is a hyperfield isomorphism. Then, for each prime integer $p, \alpha(p)= \pm q$ for some prime integer $q$, and $p=2 \Rightarrow q=2$. 
Proof. Let $K=\mathbb{Q}_{a, b}, L=\mathbb{Q}_{c, d}$. Theorem 4.4 shows that $r \mapsto \alpha(r)$ defines a group automorphism of $\mathbb{Q}^{*} / \mathbb{Q}^{* 2}$. For $r \in \mathbb{Q}^{*} / \mathbb{Q}^{* 2}$, define

$$
\mathcal{S}_{K}(r):=\left\{v \in \nu_{K, 1} \cup \nu_{K, 2}: r \notin U_{v} K^{* 2}\right\} .
$$

Note that $\mathcal{S}_{K}( \pm 1)$ is the empty set. Let $p$ be a prime. The set $\mathcal{S}_{K}( \pm p)$ is non-empty (by Lemma 4.6) and is minimal among all non-empty $\mathcal{S}_{K}(r)$. It follows that $\mathcal{S}_{L}(\alpha(p))$ is non-empty and minimal among all non-empty $\mathcal{S}_{L}(s), s \in \mathbb{Q}^{*} / \mathbb{Q}^{* 2}$, so $\alpha(p)= \pm q$, for some prime $q$. Note also that if $p=2$, then $\mathcal{S}_{K}(p)$ is a subset of $\nu_{K, 2}$. Then $\mathcal{S}_{L}( \pm q)$ is a subset of $\nu_{L, 2}$, so $q=2$.

Function fields of conics defined over local fields have been investigated by the authors in their earlier work [6]. There, the following versions of Corollaries 4.1 and 4.2 are established for local fields:

Theorem 4.10 ([6, Theorem 3.5]). Suppose $K, L$ are function fields in one variable over local fields of characteristic $\neq 2$ which are Witt equivalent via a hyperfield isomorphism $\alpha: Q(K) \rightarrow Q(L)$. Then for each $i \in\{0,1,2,3\}$ there is a uniquely defined bijection between $\mu_{K, i}$ and $\mu_{L, i}$ such that, if $v \leftrightarrow w$ under this bijection, then $\alpha$ maps $\left(1+M_{v}\right) K^{* 2} / K^{* 2}$ onto $\left(1+M_{w}\right) L^{* 2} / L^{* 2}$ and $U_{v} K^{* 2} / K^{* 2}$ onto $U_{w} L^{* 2} / L^{* 2}$ for $i \in\{0,1,2\}$ and such that $\alpha$ maps $\left(1+M_{v}\right) K^{* 2} / K^{* 2}$ onto $\left(1+M_{w}\right) L^{* 2} / L^{* 2}$ for $i=3$.

Theorem 4.11 ([6, Theorem 3.6]). Let $K \sim L$ be function fields in one variable over local fields $k$ and $\ell$ respectively, with fields of constants $k$ and $\ell$ respectively. Then $k \sim \ell$ except possibly when $k, \ell$ are both dyadic local fields. In the latter case if there exists $v \in \mu_{K, 0}$ with $K_{v}=k$ and $w \in \mu_{L, 0}$ with $L_{w}=\ell$ then $k \sim \ell$.

In view of the abovementioned results, we are able to slightly extend the results of Proposition 4.9 to the local case:

Theorem 4.12. Suppose $k, \ell$ are local fields of characteristic $\neq 2, a, b \in k^{*}$, $c, d \in \ell^{*}$. Then $k_{a, b} \sim \ell_{c, d} \Rightarrow k \sim \ell$.

Proof. By Theorem 4.11 it suffices to deal with the case where $k, \ell$ are both dyadic. Let $\alpha: Q\left(k_{a, b}\right) \rightarrow Q\left(\ell_{c, d}\right)$ be some hyperfield isomorphism. Making use of the bijection between $\mu_{K, 0}$ and $\mu_{L, 0}$ induced by $\alpha$, and arguing as in the proof of Theorem 4.4 we see that $\alpha$ induces an isomorphism between $k^{*} / k^{* 2}$ and $\ell^{*} / \ell^{* 2}$. This implies $\left[k: \hat{\mathbb{Q}}_{2}\right]=\left[\ell: \hat{\mathbb{Q}}_{2}\right]$. Thus $k \sim \ell$ iff $k, \ell$ have the same level i.e. the minimal number of summands in a representation 
of -1 as a sum of squares. The level of a dyadic local field is 1,2 or 4 . If $k$ has level 4 then $\left[k: \hat{\mathbb{Q}}_{2}\right]=\left[\ell: \hat{\mathbb{Q}}_{2}\right]$ is odd so $\ell$ has level 4 . If $k$ has level 1 then $k_{a, b}$ and consequently also $\ell_{c, d}$ has level 1 . Since $\ell$ is algebraically closed in $\ell_{c, d}$ this implies $\ell$ has level 1 .

Theorem 4.13. Suppose $k$ is a local field of characteristic $\neq 2, a, b, c, d \in$ $k^{*}$. Then $k_{a, b} \sim k_{c, d} \Rightarrow\left(\frac{a, b}{k}\right)=\left(\frac{c, d}{k}\right)$ except possibly in the case when $k$ is $p$-adic of level 1 , for some odd prime $p$.

Proof. If $k=\mathbb{C}$ there is only one quaternion algebra and the result is obvious. Otherwise, there are two quaternion algebras, one split and one non-split. Suppose $K=k_{a, b}, L=k_{c, d},\left(\frac{a, b}{k}\right)$ split, $\left(\frac{c, d}{k}\right)$ non-split. Suppose $k=\mathbb{R}$. Then $K$ is formally real and $L$ is non-real (of level 2 ), so $K \nsim L$. Suppose now that $k$ is dyadic. Suppose $K \sim L$. Fix a hyperfield isomorphism $\alpha: Q(K) \rightarrow Q(L)$. Fix $v \in \mu_{K, 0}$ with $K_{v}=k$ and let $w$ be the corresponding element of $\mu_{L, 0}$. Then $k=K_{v} \sim L_{v}$ so $\left[k: \hat{\mathbb{Q}}_{2}\right]=\left[K_{v}: \hat{\mathbb{Q}}_{2}\right]=\left[L_{w}: \hat{\mathbb{Q}}_{2}\right]$. Since $k \subseteq L_{w}$, this forces $L_{w}=k$, i.e., $\left(\frac{c, d}{k}\right)$ splits, a contradiction. Suppose now that $k$ is $p$-adic, $p \neq 2$, and $k$ has level 2. Since $k$ has level 2, we may assume $c=\pi$, where $v_{0}(\pi)=1$, and $d=-1$. Thus $\exists f, g \in L^{*}$ such that $\pi=f^{2}+g^{2}$. For each $w \in \mu_{L, 1}, w(\pi)=v_{0}(\pi)=1$ is odd, so $w\left(f^{2}\right)=w\left(g^{2}\right)<w\left(f^{2}+g^{2}\right)$, i.e., -1 is a square in $L_{w}$, for all $w \in \mu_{L, 1}$. Suppose $K \sim L$. Fix a hyperfield isomorphism $\alpha: Q(K) \rightarrow Q(L)$. Then the induced one-to-one correspondence $v \leftrightarrow w$ between $\mu_{K, 1}$ and $\mu_{L, 1}$ and the induced hyperfield isomorphisms $Q\left(K_{v}\right) \rightarrow Q\left(L_{w}\right)$ imply -1 is a square in $K_{v}$ for all $v \in \mu_{K, 1}$. Define one particular such $v$ as follows: Since $\left(\frac{a, b}{k}\right)$ splits, $K=k(x)$. Extend $v_{0}$ to $K$ by defining $v\left(\sum_{i=0}^{n} a_{i} x^{i}\right)=\min \left\{v_{0}\left(a_{i}\right): i \in\{0, \ldots, n\}\right\}$. Clearly $v \in \mu_{K, 1}$ and $K_{v}=k_{v_{0}}(x)$. But then $K_{v}$ and $k_{v_{0}}$ both have the same level, contradicting the fact that $K_{v}$ has level 1 and $k_{v_{0}}$ has level 2 .

\section{References}

[1] J.K. Arason, R. Elman, W. Jacob, Rigid elements, valuations, and realization of Witt rings. J. Algebra 110 (1987) 449-467.

[2] R. Baeza, R. Moresi, On the Witt-equivalence of fields of characteristic 2. J. Algebra 92 (1985), no. 2, 446-453.

[3] P.E. Conner, J. Hurrelbrink, Class number parity. Series in Pure Mathematics 8, World Scientific, Singapore, New Jersey, Hong Kong, 1988.

[4] P. Gładki, M. Marshall. The pp conjecture for spaces of orderings of rational conics. J. Algebra Appl. 6 (2007) 245-257.

[5] P. Gładki, M. Marshall, Witt equivalence of function fields over global fields, Trans. Amer. Math. Soc. 369 (2017), 7861-7881 
[6] P. Gładki, M. Marshall, Witt equivalence of function fields of curves over local fields, Comm. Algebra 45 (2017), 5002-5013.

[7] N. Grenier-Boley, D.W. Hoffmann, Isomorphism criteria for Witt rings of real fields. With appendix by Claus Scheiderer. Forum Math. 25 (2013) 1-18.

[8] D.K. Harrison, Witt rings. University of Kentucky Notes, Lexington, Kentucky (1970).

[9] J.L. Kleinstein, A. Rosenberg, Succinct and representational Witt rings. Pacific J. Math. 86 (1980) 99-137.

[10] M. Knebusch, Generic splitting of quadratic forms I, Proc. London Math. Soc. (3) 33 (1976) 65-93.

[11] M. Knebusch, A. Rosenberg, R. Ware, Structure of Witt rings and quotients of Abelian group rings. Amer. J. Math. 94 (1972), 119-155.

[12] P. Koprowski, Witt equivalence of algebraic function fields over real closed fields. Math. Z. 242 (2002) 323-345.

[13] T.-Y. Lam, Introduction to quadratic forms over fields. Graduate Studies in Mathematics 67 American Mathematical Society, Providence, RI, 2005.

[14] M. Marshall, Abstract Witt rings, Queen's Papers in Pure and Applied Math. 57, Queen's University, Kingston, Ontario (1980).

[15] J. Milnor, D. Husemoller, Symmetric bilinear forms. Ergebnisse der Mathematik und ihrer Grenzgebiete, Band 73. Springer-Verlag, New York-Heidelberg, 1973.

[16] R. Perlis, K. Szymiczek, P.E. Conner, R. Litherland, Matching Witts with global fields. Contemp. Math. 155 (1994) 365-378.

[17] K. Szymiczek, Matching Witts locally and globally. Math. Slovaca 41 (1991) $315-330$.

[18] K. Szymiczek, Hilbert-symbol equivalence of number fields, Tatra Mount. Math. Publ. 11 (1997), 7-16.

[19] E. Witt, Gegenbeispiel zum Normensatz. Math. Zeit. 39 (1934) 12-28.

[20] E. Witt, Theorie der quadratischen Formen in beliebigen Körpern. Journal für die reine und angewandte Mathematik 176 (1937) 31-44.

\section{CONTACT INFORMATION}

Paweł Gładki

Institute of Mathematics, University of Silesia, ul. Bankowa 14, 40-007 Katowice, Poland, and Department of Computer Science, AGH University of Science and Technology, al. Mickiewicza 30, 30-059 Kraków, Poland E-Mail(s): pawel.gladki@us.edu.pl

Received by the editors: 25.10.2018. 